\title{
Well posedness for semidiscrete fractional Cauchy problems with finite delay
}

\author{
Carlos Lizama \\ Universidad de Santiago de Chile, Facultad de Ciencias, Departamento de Matemática y Ciencia de la Computación, Las \\ Sophoras 173, Santiago, Chile. \\ e-mail: carlos.lizama@usach.cl \\ Marina Murillo-Arcila \\ BCAM - Basque Center for Applied Mathematics, Mazarredo, 14 E48009 Bilbao, Spain. \\ e-mail: mmurillo@bcamath.org
}

\begin{abstract}
We address the study of well posedness on Lebesgue spaces of sequences for the following fractional semidiscrete model with finite delay

$$
\Delta^{\alpha} u(n)=T u(n)+\beta u(n-\tau)+f(n), \quad n \in \mathbb{N}, \quad 0<\alpha \leq 1, \quad \beta \in \mathbb{R}, \quad \tau \in \mathbb{N}_{0},
$$

where $T$ is a bounded linear operator defined on a Banach space $X$ (typically a space of functions like $\left.L^{p}(\Omega), 1<p<\infty\right)$ and $\Delta^{\alpha}$ corresponds to the time discretization of the continuous Riemann-Liouville fractional derivative by means of the Poisson distribution. We characterize the existence and uniqueness of solutions in vector-valued Lebesgue spaces of sequences of the model (0.1) in terms of boundedness of the operator-valued symbol

$$
\left((z-1)^{\alpha} z^{1-\alpha} I-\beta z^{-\tau}-T\right)^{-1}, \quad|z|=1, z \neq 1
$$

whenever $0<\alpha \leq 1$ and $X$ satisfies a geometrical condition. For this purpose, we use methods from operator-valued Fourier multipliers and resolvent operator families associated to the homogeneous problem. We apply this result to show a practical and computational criterion in the context of Hilbert spaces.
\end{abstract}

Keywords: Fractional differences; Delay equations; Well-posedness; Maximal regularity; Operator-valued Fourier multiplier.

\section{Introduction}

Time delay equations appear in computational and applied contexts that have been the subject of research of many authors in the last decades. For instance, $\mathrm{Fu}$ and $\mathrm{Li}$ in [17], studied the well posedness of evolution equations with infinite delay in Lebesgue, Besov and Triebel-Lizorkin scales of vector-valued Banach spaces. Other references in this line of research are [8], [13], [15], [29] and [30]. See also the bibliography therein. In the semidiscrete case, there is also a big amount of investigation where the study of such equations naturally arises. In a first survey by H. Bateman [7] their relevance in several practical fields of interest was explained. Some of such applications appear in the theory of the compound pendulum, surges in springs and connected systems of springs, equations of Born and von Kármán and

\footnotetext{
${ }^{1}$ The first author is partially supported by CONICYT under FONDECYT grant number 1140258 and CONICYT - PIA - Anillo ACT1416.

${ }^{2}$ The second author is supported by the Basque Government through the BERC 2014-2017 program and by Spanish Ministry of Economy and Competitiveness MINECO: BCAM Severo Ochoa excellence accreditation SEV-2013-0323 and 644202 GEAGAM, H2020-MSCA-RISE-2014.
} 
elastic waves in a lattice. More recently, $\mathrm{Hu}$ and $\mathrm{Li}$ in [18] and $\mathrm{Z}$. Yu in [40] examined the qualitative behaviour of the solutions of nonlocal semidiscrete equations with delay, which concerns the distribution of the mature population of a single species. Also, in [27] Mallet-Paret studied the existence of traveling waves for semidiscrete equations that include the well-known versions of the Nagumo and KPP-Fisher equations, highlighting their practical usefulness and main differences with the continuous case.

The first studies concerning well posedness (or maximal regularity) of evolution equations using operator-valued Fourier multipliers methods are due to Amman [3] and Weis [35]. Some other authors continued this avenue of research. See for instance [4], [10], [11], [12] and [31]. More recently, methods from operator-valued Fourier multipliers were used in [23], [24], and [25] to successfully characterize the existence and uniqueness of $\ell_{p}$-solutions for discrete time fractional models.

Discrete fractional tools arise as a necessity of modelling the behavior of dynamical systems that have a non-local character in their nature, but that have been realized until now as local. The appearance of such dynamical systems concern, generally speaking, to nano-evolutions i.e. mathematical models that represent evolutions at a nano-level of interest, and which become nowadays more important because of the rapid development of nano-technologies. First studies are due to Tarasov [33, 34] in the field of nano-mechanics and physics, and to $\mathrm{Wu}$, Baleanu et.al. [36], [37] in the study of the chaotic behavior of discrete fractional models. It is worthwhile to observe that fractional differences do not only exhibit the advantages of memory effects, as the continuous case does, but they also involve fewer numerical computations, as the recent research of Wu, Baleanu and Xie [38] reveals.

In [24], the authors considered the discrete time fractional operator corresponding to the discretization, via the Poisson distribution, of the continuous Riemann-Liouville fractional derivative of order $\alpha$ defined on $\mathbb{R}_{+}$; see [22, Theorem 3.5] and Definition 2.3 below. The same discrete time fractional operator has been considered in the paper [23], where it appears in connection with the study of well posedness for semidiscrete fractional problems without delay. A variant of this problem for equations defined on $\mathbb{Z}$ was proposed in [25] for the analysis of maximal regularity of semidiscrete equations in case of unbounded operators. Numerical consequences using results of maximal regularity have been also recently studied by Kovacs, Li and Lubich in [19] and Akrivis, Li and Lubich [2].

However, none of these studies include the analysis of well posedness for semidiscrete equations with finite delay. This is a very difficult task, because the underlying sequence of operators that defines the homogeneous problem, and that completely determines the behavior of the associated non linear models is, in general, hard to find.

Nevertheless, in this paper, we have success in discovering the underlying sequence of operators and to find a complete characterization for the well posedness in Lebesgue spaces of sequences for a very general model that involves both, discrete time delays and fractional differences. Notably, our characterization is given only in terms of the data of the proposed model. In addition, our method will provide a practical and computational criterion in the setting of Hilbert spaces, that satisfies the characterization given.

More precisely, we consider the following linear fractional model with finite delay

$$
\left\{\begin{aligned}
\Delta^{\alpha} u(n) & =T u(n)+\beta u(n-\tau)+f(n), \quad n \in \mathbb{N}, \quad 0<\alpha \leq 1, \quad \beta \in \mathbb{R}, \\
u(j) & =0, \quad j=-\tau, \ldots 0, \quad \tau \in \mathbb{N}_{0},
\end{aligned}\right.
$$

where $T$ is a bounded linear operator defined on a Banach space $X$ and $f: \mathbb{N} \rightarrow X$. It is important to observe that the context of Banach spaces allows to model fractional dynamical systems that do not only involve a discrete time variable but also a continuous space variable, because in the practice $X$ is usually a space of functions like e.g. $X=L^{p}(\Omega)$ where $\Omega$ is an open subset of $\mathbb{R}^{N}$. For some examples of bounded operators $T$ in the above setting, see the recent paper [26] and references therein.

Our plan in this paper is the following: In Section 2, we introduce some basic concepts related to fractional differences and operator-valued multiplier theorems that will be later needed. In Section 3, we introduce the new concept of $\alpha^{\tau}$-resolvent sequence of bounded operators. This family of bounded operators denoted by $\left\{S_{\alpha}(n)\right\}_{n \geq \tau}$, incorporates the delay in its definition and will be crucial for obtaining an explicit representation of the solution for the fractional equation (1.1). We succeed in showing that the sequence

$$
u(n+1)=S_{\alpha}(n+1) u(0)+\beta \sum_{j=1}^{\tau} S_{\alpha}(n-\tau+j) u(-j)+\left(S_{\alpha} * f\right)(n), n \in \mathbb{N}_{0},
$$


solves (1.1) with initial condition $u(0)=x_{0}$ and a finite number of delays $u(j)=x_{j}, j=-\tau, \ldots,-1$.

Finally, in Section 4 we address the study of well posedness for (1.1). It is notable that we provide a characterization of this fact in terms of the $R$-boundedness of the operator-valued symbol associated to the abstract model.

More concretely, we show that when $X$ is a $U M D$ space and some conditions on the resolvent family such as $\sup _{n \in \mathbb{N}_{0}}\left\|S_{\alpha}(n)\right\|<\infty$ are satisfied, the well posedness of (1.1) is equivalent to the $R$ boundedness of the sets $\left\{z^{1-\alpha}(z-1)^{\alpha} M(z):|z|=1, z \neq 1\right\}$ and $\left\{z^{-\tau} M(z):|z|=1, z \neq 1\right\}$, where $M(z):=\left(z^{1-\alpha}(z-1)^{\alpha}-T-\beta z^{-\tau}\right)^{-1}$. Our characterization provides new insights in the study of the behavior and regularity of the solutions of a wide class of discrete time equations with delay. In particular, it provides a useful a priori estimate for the solutions, namely, there exists a constant $C>0$ (independent of $\left.f \in \ell_{p}\left(\mathbb{N}_{0} ; X\right)\right)$ such that the following inequality holds

$$
\left\|\Delta^{\alpha} u\right\|_{\ell_{p}\left(\mathbb{N}_{0} ; X\right)}+\|u\|_{\ell_{p}\left(\mathbb{N}_{0} ; X\right)}+\|T u\|_{\ell_{p}\left(\mathbb{N}_{0} ; X\right)} \leq C\|f\|_{\ell_{p}\left(\mathbb{N}_{0} ; X\right)} .
$$

Concerning this last estimate, it has been recently highlighted by Akrivis, Li and Lubich [2] that the combination of discrete maximal regularity and energy estimates is very useful to derive optimal-order error bounds for the time-discrete approximation of quasilinear parabolic equations by backward difference formulas. As pointed out by $\mathrm{Li}$ in [20], this estimate can be regarded as the stability of the parabolic projection onto the finite element space. These results are required for instance in [21] to establish optimal $L^{p}\left((0 ; T) ; L^{q}\right)$ error estimates of finite element methods for parabolic equations.

In order to illustrate our results, we finish this paper showing a practical criterion that ensures, under some assumptions on the operator $T$, the well posedness of (1.1) when $X$ is a Hilbert space. More concretely, we prove the folowing: If $X$ is a Hilbert space and $T \in \mathcal{B}(X)$ satisfies the following condition on the size of their norm

$$
|| T||<w_{\alpha, \beta, \tau}:=\min _{|z|=1}\left|f_{\alpha, \beta, \tau}(z)\right|<1 \quad \text { where } \quad f_{\alpha, \beta, \tau}(z):=z^{1-\alpha}(z-1)^{\alpha}-\beta z^{-\tau},
$$

then (1.1) has maximal $\ell_{p}$-regularity.

Finally, we remark that the study done in this paper was motivated by recent studies on semidiscrete mathematical models that prove that they can serve as a new microstructural basis for fractional nonlocal continuum mechanics and physics [33], [34]. Fractional order semidiscrete equations can be also used to formulate adequate models in nanomechanics [34], [36] and therefore further studies in this class of semidiscrete equations deserve to be investigated. Our contribution in this paper provides a new qualitative advance in this line of research, that incorporates tools from operator theory and allows the analysis of well posedness for a very general but still simple model. The study of more complex dynamical systems that include unbounded operators is still an open problem. This task will be the objective of forthcoming works.

\section{Preliminaries}

We denote by $\ell_{p}\left(\mathbb{N}_{0} ; X\right), 1 \leq p<\infty$, the Banach space consisting of all vector-valued and $p$-summable sequences $f: \mathbb{N}_{0} \rightarrow X$. The forward Euler operator $\Delta: \ell_{p}\left(\mathbb{N}_{0} ; X\right) \rightarrow \ell_{p}\left(\mathbb{N}_{0} ; X\right)$ is defined by

$$
\Delta f(t):=f(t+1)-f(t), \quad t \in \mathbb{N}_{0} .
$$

We also denote $\Delta^{0} \equiv I$, where $I$ is the identity operator. Observe that $\Delta$ is a bounded operator with $\|\Delta\| \leq 2$.

The following definition was presented in [23, Formula 2.2] in the context of abstract Cauchy problems on time difference equations. It corresponds to an special case of fractional sum proposed by Atici and Eloe in [6]. It has been recently observed that it coincides with the notion of Cesáro sums of order $\alpha>0$ and possess an strong connection with certain algebra homomorphisms.

Definition 2.1. Let $\alpha>0$ be given and $f: \mathbb{N}_{0} \rightarrow X$. We define the fractional sum of order $\alpha$ as follows

$$
\Delta^{-\alpha} f(n)=\sum_{k=0}^{n} k^{\alpha}(n-k) f(k), \quad n \in \mathbb{N}_{0},
$$


where

$$
k^{\alpha}(j)=\frac{\Gamma(\alpha+j)}{\Gamma(\alpha) \Gamma(j+1)}, \quad j \in \mathbb{N}_{0} .
$$

Remark 2.2. We note that allowing $\alpha<0$ in the above definition, we get the concept of fractional difference in the sense of Grünwald-Letnikov. Observe that this definition corresponds to discretization by means of the backward Euler operator, see the comments after Definition 2.1 in [23].

The following notion is similar to the concept of a fractional derivative in the sense of RiemannLiouville, see [28] and [5]. It is interesting to observe that it coincides with the sampling by means of the Poisson distribution of the continuous fractional difference of the same order. See [23, Theorem 3.5].

Definition 2.3. Given $f: \mathbb{N}_{0} \rightarrow X$, the fractional difference operator of order $\alpha>0$ (in the sense of Riemann-Liouville) is defined by

$$
\Delta^{\alpha} f(n):=\Delta^{m} \circ \Delta^{-(m-\alpha)} f(n), \quad n \in \mathbb{N}_{0},
$$

where $m-1<\alpha<m, m=\lceil\alpha\rceil$.

We finish this section recalling several well-known concepts and notations concerning the discrete setting that will be useful in the development of this work.

Given $f, g \in \ell_{p}\left(\mathbb{N}_{0} ; X\right)$ we define the convolution product

$$
(f * g)(n):=\sum_{j=0}^{n} f(n-j) g(j), \quad n \in \mathbb{N} .
$$

We now recall the notions of $Z$-transform and discrete time Fourier Transform.

1. The $Z$-transform of a vector-valued sequence $f: \mathbb{N}_{0} \rightarrow X$ is defined by

$$
\widetilde{f}(z):=\sum_{j=0}^{\infty} z^{-j} f(j)
$$

where $z$ denotes a complex number. Note that convergence of the series is given for $|z|>R$ with $R$ sufficiently large.

2. The discrete time Fourier transform of a vector valued sequence $f: \mathbb{Z} \rightarrow X$ is defined by

$$
\widehat{f}(z):=\sum_{j=-\infty}^{\infty} z^{-j} f(j), \text { where } z=e^{i t}, \quad t \in(-\pi, \pi),
$$

whenever it exists.

Remark 2.4. Note that the $Z$-transform around the unit circle in the complex plane corresponds to the discrete time Fourier transform, whenever both exists.

The following Fourier multiplier theorem for operator valued symbols is due to S. Blunck [9]. This theorem is stated for the UMD class of Banach spaces. For more information about these spaces see [3, Section III.4.3-III.4.5]). We will first recall the notion of $R$-bounded sets in the space $\mathcal{B}(X, Y)$ of bounded linear operators from $X$ into $Y$ endowed with the uniform operator topology.

Definition 2.5. Let $X$ and $Y$ be Banach spaces. A subset $\mathcal{T}$ of $\mathcal{B}(X, Y)$ is called $R$-bounded if there is a constant $c \geq 0$ such that

$$
\left\|\left(T_{1} x_{1}, \ldots, T_{n} x_{n}\right)\right\|_{R} \leq c\left\|\left(x_{1}, \ldots, x_{n}\right)\right\|_{R},
$$

for all $T_{1}, \ldots, T_{n} \in \mathcal{T}, x_{1}, \ldots, x_{n} \in X, n \in \mathbb{N}$, where

$$
\left\|\left(x_{1}, \ldots, x_{n}\right)\right\|_{R}:=\frac{1}{2^{n}} \sum_{\epsilon_{j} \in\{-1,1\}^{n}}\left\|\sum_{j=1}^{n} \epsilon_{j} x_{j}\right\|,
$$

for $x_{1}, \ldots, x_{n} \in X$. 
For more information about $R$-bounded sets and their properties see [1, Section 2.2] and [14]. In what follows we denote $\mathbb{T}:=(-\pi, \pi) \backslash\{0\}$.

Theorem 2.6. [9, Theorem 1.3] Let $p \in(1, \infty)$ and let $X$ be a $U M D$ space. Let $M: \mathbb{T} \rightarrow \mathcal{B}(X)$ be differentiable and such that the set

$$
\left\{M(t),(z-1)(z+1) M^{\prime}(t): z=e^{i t}, t \in \mathbb{T}\right\}
$$

is $R$-bounded. Then there is an operator $T_{M} \in \mathcal{B}\left(l_{p}(\mathbb{Z} ; X)\right)$ such that

$$
\left.\widehat{\left(T_{M} f\right.}\right)(z)=M(t) \widehat{f}(z), \text { for all } z=e^{i t}, t \in \mathbb{T} .
$$

The converse of Blunck's theorem also holds but the Banach space $X$ does not need to be a $U M D$ space.

Theorem 2.7. [9] Let $p \in(1, \infty)$ and let $X$ be a Banach space. Let $M: \mathbb{T} \rightarrow \mathcal{B}(X)$ be an operator valued function. Suppose that there is an operator $T_{M} \in \mathcal{B}\left(l_{p}(\mathbb{Z} ; X)\right)$ such that $(2.3)$ holds. Then the set

$$
\{M(t): t \in \mathbb{T}\}
$$

is R-bounded.

\section{3. $\alpha^{\tau}$-resolvent families}

In this section, we introduce an operator theoretical method to study the linear fractional difference problem

$$
\begin{cases}\Delta^{\alpha} u(n) & =T u(n)+\beta u(n-\tau)+f(n), \quad n \in \mathbb{N}, \tau \in \mathbb{N}_{0}, \beta \in \mathbb{R} \\ u(i) & =x_{i}, i=-\tau, \ldots, 0\end{cases}
$$

where $T \in \mathcal{B}(X)$. More concretely, we study the existence and uniqueness of solutions of equation (3.1) for $0<\alpha \leq 1$ and $T \in \mathcal{B}(X)$. To proceed, we need to introduce the notion of an $\alpha^{\tau}$ - resolvent sequence of operators that allows us to obtain an explicit representation for the solution of equation (3.1) by means of a kind of variation of parameters formula. We begin with the following definition.

Definition 3.1. Let $T$ be a bounded operator defined on a Banach space $X, 0<\alpha \leq 1$ and $\tau \in \mathbb{N}_{0}$. $T$ is called the generator of an $\alpha^{\tau}$-resolvent sequence if there exists a sequence of bounded and linear operators $\left\{S_{\alpha}(n)\right\}_{n \geq-\tau} \subset \mathcal{B}(X)$ that satisfies the following properties

(i) $S_{\alpha}(0)=I, S_{\alpha}(-1)=0, S_{\alpha}(-2)=0, \ldots, S_{\alpha}(-\tau)=0$,

(ii) $S_{\alpha}(n+1)=k^{\alpha}(n+1) I+T\left(k^{\alpha} * S_{\alpha}\right)(n)+\beta\left(k^{\alpha} * S_{\alpha}^{\tau}\right)(n)$ for all $n \in \mathbb{N}_{0}$,

where we denote $S_{\alpha}^{\tau}(n):=S_{\alpha}(n-\tau)$.

Remark 3.2. Note that Definition 3.1 corresponds to the resolvent sequence defined in [23] when $\beta=0$.

Example 3.3. Suppose that $\left\{z^{1-\alpha}(z-1)^{\alpha}-\beta z^{-\tau}\right\}_{z \in C} \in \rho(T)$, where $\rho(T)$ denotes the resolvent set of $T$ and $C$ is a circle centered at the origin of the complex plane that encloses all singularities of $z^{n}\left(z^{1-\alpha}(z-1)^{\alpha}-\beta z^{-\tau}-T\right)^{-1}$ in its interior. Then the formula

$$
S_{\alpha}(n):= \begin{cases}\frac{1}{2 \pi i} \int_{C} z^{n}\left(z^{1-\alpha}(z-1)^{\alpha}-\beta z^{-\tau}-T\right)^{-1} d z & n \in \mathbb{N}, \\ 0 & n=-\tau, \ldots, 0,\end{cases}
$$

defines an $\alpha^{\tau}$ - resolvent sequence of operators with generator $T$.

Before obtaining an explicit solution of problem (3.1) in terms of the $\alpha^{\tau}$-resolvent family we need the following Lemma stated in [23, Lemma 3.6]. 
Lemma 3.4. Let $0<\alpha<1, a: \mathbb{N}_{0} \rightarrow \mathbb{C}$ and $S: \mathbb{N}_{0} \rightarrow X$ be given. Then

$$
\Delta^{\alpha}(a * S)(n)=\left(a * \Delta^{\alpha} S\right)(n)+S(0) a(n+1), \quad n \in \mathbb{N}_{0},
$$

holds.

Now we are ready to prove the main result of this section.

Theorem 3.5. Let $f: \mathbb{N}_{0} \rightarrow X$ be given. Given $0<\alpha \leq 1$ the unique solution of (3.1) with initial conditions $u(j)=x_{j}, j=-\tau, \ldots, 0$ can be represented by

$$
u(n+1)=S_{\alpha}(n+1) u(0)+\beta \sum_{j=1}^{\tau} S_{\alpha}(n-\tau+j) u(-j)+\left(S_{\alpha} * f\right)(n), n \in \mathbb{N}_{0} .
$$

Proof. To prove the assertion we define

$$
v(n)= \begin{cases}S_{\alpha}(n) u(0)+\beta \sum_{j=1}^{\tau} S_{\alpha}(n-1-\tau+j) u(-j), & n \geq 1, \\ x_{n} & n=-\tau, \ldots, 0 .\end{cases}
$$

Let us check that $v$ is a solution of (3.1) with $f=0$. Indeed, given $n \in \mathbb{N}$ we have

$$
\Delta^{\alpha} v(n+1)=\Delta^{\alpha} S_{\alpha}(n+1) u(0)+\beta \sum_{j=1}^{\tau} \Delta^{\alpha} S_{\alpha}(n-\tau+j) u(-j), n \in \mathbb{N}_{0},
$$

where, by definition

$$
\Delta^{\alpha} S_{\alpha}(n)=\Delta^{\alpha} k^{\alpha}(n)+T \Delta^{\alpha}\left(k^{\alpha} * S_{\alpha}\right)(n-1)+\beta \Delta^{\alpha}\left(k^{\alpha} * S_{\alpha}^{\tau}\right)(n-1), n \in \mathbb{N} .
$$

Note that $\Delta^{\alpha} k^{\alpha}(n)=0$, therefore using Lemma 3.4 we obtain

$$
\begin{aligned}
\Delta^{\alpha} S_{\alpha}(j+1) & =T \Delta^{\alpha}\left(k^{\alpha} * S_{\alpha}\right)(j)+\beta \Delta^{\alpha}\left(k^{\alpha} * S_{\alpha}^{\tau}\right)(j) \\
& =T\left(\Delta^{\alpha} k^{\alpha} * S_{\alpha}\right)(j)+k^{\alpha}(0) T S_{\alpha}(j+1)+\beta\left(\Delta^{\alpha} k^{\alpha} * S_{\alpha}^{\tau}\right)(j)+k^{\alpha}(0) \beta S_{\alpha}^{\tau}(j+1) \\
& =T S_{\alpha}(j+1)+\beta S_{\alpha}^{\tau}(j+1),
\end{aligned}
$$

for all $j \in \mathbb{N}_{0}$. Hence $\Delta^{\alpha} S_{\alpha}(n)=T S_{\alpha}(n)+\beta S_{\alpha}^{\tau}(n)$ and we have for all $n \in \mathbb{N}$

$$
\begin{aligned}
\Delta^{\alpha} v(n) & =T S_{\alpha}(n) u(0)+\beta S_{\alpha}^{\tau}(n) u(0)+\beta \sum_{j=1}^{\tau}\left(T S_{\alpha}(n-\tau+j-1)+\beta S_{\alpha}^{\tau}(n-\tau-j-1)\right) u(-j) \\
& =T v(n)+\beta v(n-\tau) .
\end{aligned}
$$

Then, we conclude that (3.4) is the solution of the equation (3.1) with $f=0$. Now, let us define

$$
w(n)= \begin{cases}\left(S_{\alpha} * f\right)(n-1), & n \geq 1, \\ 0 & n=-\tau, \ldots, 0 .\end{cases}
$$

We get using Lemma 3.4 and 3.6 that

$$
\begin{aligned}
\Delta^{\alpha} w(n) & =\Delta^{\alpha}\left(S_{\alpha} * f\right)(n-1)=T\left(S_{\alpha} * f\right)(n-1)+\beta\left(S_{\alpha}^{\tau} * f\right)(n-1)+f(n) \\
& =T w(n)+\beta \sum_{j=0}^{n-1} S_{\alpha}(n-1-\tau-j) f(j)+f(n)=T w(n)+\beta w(n-\tau)+f(n),
\end{aligned}
$$

for all $n \in \mathbb{N}$. Observe that the last equality holds since $S_{\alpha}(n)=0$, for all $n=-\tau, \ldots, 0$. Therefore, $w$ solves (3.1) with homogeneous initial conditions $w(j)=0, j=-\tau, \ldots, 0$.. We conclude that $u=v+w$ solves (3.1). Indeed,

$$
\begin{aligned}
\Delta^{\alpha} u(n) & =\Delta^{\alpha} v(n)+\Delta^{\alpha} w(n)=T v(n)+\beta v(n-\tau)+T w(n)+\beta w(n-\tau)+f(n) \\
& =T u(n)+\beta u(n-\tau)+f(n), \quad n \geq 1
\end{aligned}
$$

and $u(j)=x_{j}$, for all $j=-\tau, \ldots, 0$ and the theorem is proved. 


\section{A characterization of maximal $l_{p}$-regularity}

Let $T \in \mathcal{B}(X)$ be given and $f: \mathbb{N}_{0} \rightarrow X$ be a vector valued sequence. In this section, we consider the discrete time evolution equation of fractional order with finite delay $\tau \in \mathbb{N}_{0}$ :

$$
\left\{\begin{aligned}
\Delta^{\alpha} u(n) & =T u(n)+\beta u(n-\tau)+f(n), \quad n \in \mathbb{N}, \\
u(j) & =0, \quad j=-\tau, \ldots 0 .
\end{aligned}\right.
$$

By Theorem 3.5, the solution of equation (4.1) can be represented by

$$
u(n)=\left(S_{\alpha} * f\right)(n-1), \quad n \in \mathbb{N}, \quad 0<\alpha \leq 1 .
$$

Also note that, by Lemma 3.4,

$$
\Delta^{\alpha} u(n)=T\left(S_{\alpha} * f\right)(n-1)+\beta\left(S_{\alpha}^{\tau} * f\right)(n-1)+f(n) \quad 0<\alpha \leq 1 .
$$

Definition 4.1. Let $1<p<\infty, 0<\alpha \leq 1$ and $T \in \mathcal{B}(X)$ be given. We say that equation (4.1) has maximal $l_{p}$-regularity if $f \in l_{p}\left(\mathbb{N}_{0} ; X\right)$ implies $u, \Delta^{\alpha} u \in l_{p}\left(\mathbb{N}_{0} ; X\right)$.

Observe that in view of (4.2), the notion of maximal $\ell_{p}$-regularity is equivalent to have that

$$
\left(\mathcal{K}_{\alpha} f\right)(n):=T \sum_{j=0}^{n} S_{\alpha}(n-j) f(j) \quad \text { and } \quad\left(\mathcal{P}_{\alpha} f\right)(n):=\sum_{j=0}^{n} S_{\alpha}(n-j-\tau) f(j)=\left(S_{\alpha}^{\tau} * f\right)(n)
$$

define bounded operators $\mathcal{K}_{\alpha}, \mathcal{P}_{\alpha} \in \mathcal{B}\left(l_{p}\left(\mathbb{N}_{0} ; X\right)\right)$ for some $p \in(1, \infty)$.

Remark 4.2. Note that if (4.1) has maximal $\ell_{p}$-regularity then, by choosing $f(j)=1$ for $j=0$ and 0 otherwise, we obtain that the associated $\alpha^{\tau}$-resolvent family must be $p$-summable.

In what follows we will need the following hypothesis:

$$
(H)_{\alpha} \sup _{n \in \mathbb{N}_{0}}\left\|S_{\alpha}(n)\right\|<\infty \text { and }(z-1)^{\alpha} z^{1-\alpha} I-\beta z^{-\tau}-T \text { is invertible for all }|z|=1, z \neq 1
$$

We denote $\mathbb{D}:=\{z \in \mathbb{C}:|z| \leq 1\}$ and we define the set

$$
\Omega_{\alpha, \beta, \tau}:=\left\{z \in \mathbb{C}: z=(w-1)^{\alpha} w^{1-\alpha}-\beta w^{-\tau}, w \in \partial \mathbb{D}, w \neq 1\right\} .
$$

Theorem 4.3. Let $0<\alpha \leq 1, p>1$ and $X$ be a $U M D$ space. Let $T \in \mathcal{B}(X)$ be such that $(H)_{\alpha}$ holds. Then the following assertions are equivalent.

(i) Equation (4.1) has maximal $l_{p}$-regularity.

(ii) The sets $\left\{z^{1-\alpha}(z-1)^{\alpha}\left(z^{1-\alpha}(z-1)^{\alpha}-T-\beta z^{-\tau}\right)^{-1}:|z|=1, z \neq 1\right\}$ and

$$
\left\{z^{-\tau}\left(z^{1-\alpha}(z-1)^{\alpha}-T-\beta z^{-\tau}\right)^{-1}:|z|=1, z \neq 1\right\} \text { are } R \text {-bounded. }
$$

Proof. We first prove that (ii) $\Longrightarrow$ (i). Let us define $N(t)=z^{1-\alpha}(z-1)^{\alpha}\left(z^{1-\alpha}(z-1)^{\alpha}-T-\beta z^{-\tau}\right)^{-1}$ and $S(t)=z^{-\tau}\left(z^{1-\alpha}(z-1)^{\alpha}-T-\beta z^{-\tau}\right)^{-1}$. We write $z=e^{i t}$ and denoting $f_{\alpha}(t)=e^{i t}\left(1-e^{-i t}\right)^{\alpha}$ we have $N(t)=f_{\alpha}(t)\left(f_{\alpha}(t)-T-\beta e^{-i t \tau}\right)^{-1}$ and $S(t)=e^{-i t \tau}\left(f_{\alpha}(t)-T-\beta e^{-i t \tau}\right)^{-1}$. Now, a calculation gives

$$
\begin{aligned}
N^{\prime}(t) & =f_{\alpha}^{\prime}(t)\left(f_{\alpha}(t)-T-\beta e^{-i t \tau}\right)^{-1}-\left(f_{\alpha}(t)-T-\beta e^{-i t \tau}\right)^{-2}\left(f_{\alpha}^{\prime}(t)+i \tau \beta e^{-i t \tau}\right) f_{\alpha}(t) \\
S^{\prime}(t) & =-i \tau e^{-i t \tau}\left(f_{\alpha}(t)-T-\beta e^{-i t \tau}\right)^{-1}-\left(f_{\alpha}(t)-T-\beta e^{-i t \tau}\right)^{-2}\left(f_{\alpha}^{\prime}(t)+i \tau \beta e^{-i t \tau}\right) e^{-i t \tau},
\end{aligned}
$$


where $f_{\alpha}^{\prime}(t)=i f_{\alpha}(t)+\frac{i \alpha}{e^{i \alpha}-1} f_{\alpha}(t)=i f_{\alpha}(t)+\frac{i \alpha}{e^{i t}-1} f_{\alpha}(t)$. Therefore,

$$
N^{\prime}(t)=i N(t)+\frac{i \alpha}{e^{i t}-1} N(t)-i N(t)^{2}-\frac{i \alpha}{e^{i t}-1} N(t)^{2}-i \tau N(t) S(t),
$$

and

$$
S^{\prime}(t)=-i \tau S(t)-i N(t) S(t)-\frac{i \alpha}{e^{i t}-1} N(t) S(t)-i \tau S(t)^{2}
$$

We finally get

$$
\begin{aligned}
(z-1)(z+1) N^{\prime}(t) & =[i \alpha(z+1)(z-1)+i \alpha(z+1)] N(t)-[i(z-1)(z+1)+i \alpha(z+1)] N(t)^{2} \\
& -i \tau(z+1)(z-1) N(t) S(t)
\end{aligned}
$$

and

$$
\begin{aligned}
(z-1)(z+1) S^{\prime}(t) & =-i \tau(z-1)(z+1) S(t)-i(z+1)(z-1) N(t) S(t)-i \alpha(z+1) N(t) S(t) \\
& -i \tau(z+1)(z-1) S(t)^{2},
\end{aligned}
$$

where $z=e^{i t}, t \in(-\pi, \pi)$. We conclude from [1, Proposition 2.2.5] that the sets

$$
\left\{(z-1)(z+1) N^{\prime}(t): z=e^{i t}, t \in \mathbb{T}\right\} \quad \text { and } \quad\left\{(z-1)(z+1) S^{\prime}(t): z=e^{i t}, t \in \mathbb{T}\right\}
$$

are $R$-bounded. Therefore, by Theorem 2.6 there exist operators $T_{\alpha}, U_{\alpha} \in \mathcal{B}\left(l_{p}(\mathbb{Z} ; X)\right)$ such that

$$
\begin{aligned}
& \widehat{\left(T_{\alpha} f\right)}(z)=N(t) \widehat{f}(z), \quad z=e^{i t}, \quad t \in \mathbb{T}, \\
& \widehat{\left(U_{\alpha} f\right)}(z)=S(t) \widehat{f}(z), \quad z=e^{i t}, \quad t \in \mathbb{T},
\end{aligned}
$$

for all $f \in l^{p}(\mathbb{Z} ; X)$. From the identity

$$
T\left(z^{1-\alpha}(z-1)^{\alpha}-T-\beta z^{-\tau}\right)^{-1}=\left(z^{1-\alpha}(z-1)^{\alpha}-\beta z^{-\tau}\right)\left(z^{1-\alpha}(z-1)^{\alpha}-T-\beta z^{-\tau}\right)^{-1}-I
$$

and (4.4) we obtain that the left hand side in the identity

$$
T\left(z^{1-\alpha}(z-1)^{\alpha}-T-\beta z^{-\tau}\right)^{-1} \hat{f}(z)=\left(z^{1-\alpha}(z-1)^{\alpha}-\beta z^{-\tau}\right)\left(z^{1-\alpha}(z-1)^{\alpha}-T-\beta z^{-\tau}\right)^{-1} \hat{f}(z)-I \hat{f}(z),
$$

defines a bounded operator on $l^{p}(\mathbb{Z} ; X)$ given by $R_{\alpha} f(n)=T_{\alpha} f(n)-\beta U_{\alpha} f(n)-f(n)$.

For $f \in l^{p}(\mathbb{Z} ; X)$ we define the operator

$$
K_{\alpha} f(n)=\left\{\begin{array}{r}
T\left(S_{\alpha} * f\right)(n) \quad n \in \mathbb{N}, \\
0 \quad \text { otherwise. }
\end{array}\right.
$$

Note that the $Z$-transform of $S_{\alpha}(n)$ exists by hypothesis (4.3) and it satisfies

$$
\left((z-1)^{\alpha} z^{1-\alpha}-T-\beta z^{-\tau}\right) \widetilde{S}_{\alpha}(z)=z I,
$$

for all $|z|>1$. By hypothesis $(H)_{\alpha}$, the left hand side is invertible for $|z|=1, z \neq 1$ and therefore, in view of (4.6), the discrete Fourier transform of $K_{\alpha} f(n-1)$ coincides with $R_{\alpha} f(n)$ for all $n \in \mathbb{N}$, by uniqueness. Moreover, we define

$$
P_{\alpha} f(n)=\left\{\begin{array}{r}
\left(S_{\alpha}^{\tau} * f\right)(n) \quad n \in \mathbb{N}, \\
0 \text { otherwise, }
\end{array}\right.
$$

and considering the second equation in (4.4) we have that the discrete Fourier transform of $P_{\alpha} f(n-1)$ coincides with $U_{\alpha} f(n)$ for all $n \in \mathbb{N}$, by uniqueness. It proves (i). 
Conversely, suppose (i). Let $f \in l^{p}(\mathbb{Z} ; X)$ be given and define

$$
K_{\alpha} f(n)=\left\{\begin{array}{c}
\mathcal{K}_{\alpha} f(n) \quad n \in \mathbb{N}_{0}, \\
0 \text { otherwise, }
\end{array}\right.
$$

and

$$
P_{\alpha} f(n)=\left\{\begin{array}{c}
\mathcal{P}_{\alpha} f(n) \quad n \in \mathbb{N}_{0}, \\
0 \quad \text { otherwise. }
\end{array}\right.
$$

Let

$$
T_{\alpha} f(n):=K_{\alpha} f(n-1)+f(n), \quad \text { and } \quad U_{\alpha} f(n):=P_{\alpha} f(n-1), \quad n \in \mathbb{Z} .
$$

Then, for $z=e^{i t}, t \in(-\pi, \pi)$ we have

$$
\begin{aligned}
\widehat{T_{\alpha} f}(z) & =\sum_{j \in \mathbb{Z}} z^{-j} T_{\alpha} f(j)=\sum_{j=1}^{\infty} z^{-j} K_{\alpha} f(j-1)+\sum_{j \in \mathbb{Z}} z^{-j} f(j) \\
& =z^{-1} \sum_{j=0}^{\infty} z^{-j} K_{\alpha} f(j)+\sum_{j \in \mathbb{Z}} z^{-j} f(j) \\
& =z^{-1} \sum_{j=0}^{\infty} z^{-j} \mathcal{K}_{\alpha} f(j)+\widehat{f}(z) .
\end{aligned}
$$

By hypothesis $\left(H_{\alpha}\right),(4.8)$ and the analytic continuation principle we have that the $Z$-transform $\widetilde{S}_{\alpha}(z)$ exists for $|z|=1, z \neq 1$ and therefore

$$
\widehat{T_{\alpha} f}(z)=z^{-1} T \widehat{\mathbf{S}}_{\alpha}(z) \widehat{f}(z)+\widehat{f}(z),
$$

where

$$
\mathbf{S}_{\alpha}(n)=\left\{\begin{array}{c}
S_{\alpha}(n) \quad n \in \mathbb{N}_{0}, \\
0 \text { otherwise. }
\end{array}\right.
$$

Hence, (4.8) and (4.6) imply

$$
\begin{aligned}
\widehat{T_{\alpha} f}(z) & =T\left(z^{1-\alpha}(z-1)^{\alpha}-\beta z^{-\tau}-T\right)^{-1} \widehat{f}(z)+\widehat{f}(z) \\
& =\left(z^{1-\alpha}(z-1)^{\alpha}-\beta z^{-\tau}\right)\left(z^{1-\alpha}(z-1)^{\alpha}-\beta z^{-\tau}-T\right)^{-1} \widehat{f}(z)-\widehat{f}(z)+\widehat{f}(z) \\
& =(N(t)-\beta S(t)) \widehat{f}(z) .
\end{aligned}
$$

On the other hand,

$$
\widehat{U_{\alpha} f}(z)=z^{-1} \sum_{j=0}^{\infty} z^{-j} \mathcal{P}_{\alpha} f(j)=z^{-1} z^{-\tau} \widehat{\mathbf{S}}_{\alpha}(z) \widehat{f}(z)
$$

Hence, (4.8) implies

$$
\widehat{U_{\alpha} f}(z)=S(t) \widehat{f}(z) .
$$

Then, $N(t)$ and $S(t)$ are $R$-bounded as a consequence of Theorem 2.7.

Remark 4.4. In case that $X$ is a Hilbert space, condition $(i i)$ of Theorem 4.3 can be replaced by $(i i)^{\prime}$

$$
\begin{aligned}
& \sup _{|z|=1, z \neq 1}\left\|z^{1-\alpha}(z-1)^{\alpha}\left(z^{1-\alpha}(z-1)^{\alpha}-T-\beta z^{-\tau}\right)^{-1}\right\|<\infty \quad \text { and } \\
& \sup _{|z|=1, z \neq 1}\left\|z^{-\tau}\left(z^{1-\alpha}(z-1)^{\alpha}-T-\beta z^{-\tau}\right)^{-1}\right\|<\infty
\end{aligned}
$$


Corollary 4.5. If the hypothesis of Theorem 4.3 hold, then $u, \Delta^{\alpha} u, T u \in \ell_{p}\left(\mathbb{N}_{0} ; X\right)$ and there exists a constant $C>0$ (independent of $f \in \ell_{p}\left(\mathbb{N}_{0} ; X\right)$ ) such that the following inequality holds

$$
\left\|\Delta^{\alpha} u\right\|_{\ell_{p}\left(\mathbb{N}_{0} ; X\right)}+\|u\|_{\ell_{p}\left(\mathbb{N}_{0} ; X\right)}+\|T u\|_{\ell_{p}\left(\mathbb{N}_{0} ; X\right)} \leq C\|f\|_{\ell_{p}\left(\mathbb{N}_{0} ; X\right)} .
$$

We now illustrate this theorem with the following examples.

Example 4.6. Let us consider the simple scalar difference equation studied in [16, Section 2.5] that can be labeled into the scheme of (3.1)

$$
u(n+2)-u(n+1)=-\lambda u(n)+f(n), \quad n \in \mathbb{N}_{0},
$$

where $\lambda>0$ and $u(1)=0, u(0)=0$. The explicit solution of (4.14) with $f=0$ has the form:

$$
u(n)=\lambda_{1}^{n}\left[a_{1}+a_{2}\left(\frac{\lambda_{2}}{\lambda_{1}}\right)^{n}\right] .
$$

In our context, this equation can be reformulated as follows

$$
\Delta u(n)=-\lambda u(n-1)+f(n-1) .
$$

where $u(0)=0$ and $u(-1)=0$. The autonomous version of (4.15) was studied in [32]. Observe that equation (4.14) follows the scheme of (3.1) with $\alpha=1, T=0, \tau=1$ and $\beta=-\lambda$. To study (4.15) we use Theorem 4.3. We first compute the family $\left\{S_{\alpha}(n)\right\}_{n \geq-1}$ that defines the solution $u$ of (4.15). Indeed, using the inverse $Z$-transform we get

$$
\begin{aligned}
S_{\alpha}(n) & =\frac{1}{2 \pi i} \int_{C} z^{n-1} z\left(z-1+\lambda z^{-1}\right)^{-1} d z=\frac{1}{2 \pi i} \int_{C} \frac{z^{n+1}}{z(z-1)+\lambda} d z=\frac{1}{2 \pi i} \int_{C} \frac{z^{n+1}}{\left(z-\lambda_{1}\right)\left(z-\lambda_{2}\right)} d z \\
& =\left(\lambda_{1}{ }^{n+1}-\lambda_{2}{ }^{n+1}\right) \frac{1}{\lambda_{1}-\lambda_{2}}=\lambda_{1}{ }^{n+1}\left(1-\left(\frac{\lambda_{2}}{\lambda_{1}}\right)^{n+1}\right) \frac{1}{\lambda_{1}-\lambda_{2}},
\end{aligned}
$$

where $C$ is a circle centered at the origin of the complex plane that encloses all singularities of $z^{n}(z-1+$ $\left.\lambda z^{-1}\right)^{-1}$ in its interior, $\lambda_{1}=\frac{1+\sqrt{1-4 \lambda}}{2}$ and $\lambda_{2}=\frac{1-\sqrt{1-4 \lambda}}{2}$. As a consequence, $\sup _{n \in \mathbb{N}_{0}}\left\|S_{\alpha}(n)\right\|<\infty$ and then (4.3) holds whenever $\left|\lambda_{2}\right| \leq\left|\lambda_{1}\right|<1$, that is, for all $0<\lambda<1$. Moreover, in this case, we have that

$$
\sup _{|z|=1}\left|\frac{1}{z^{2}-z+\lambda}\right| \leq \sup _{|z|=1} \frac{1}{\left|z-\lambda_{1}\right|\left|z-\lambda_{2}\right|} \leq \frac{1}{\left(|1-| \lambda_{1}||\right)\left(|1-| \lambda_{2}||\right)}<\infty
$$

and then the sets $\left\{(z-1)\left((z-1)+\lambda z^{-1}\right)^{-1}:|z|=1, z \neq 1\right\}$ and $\left\{z^{-1}\left((z-1)+\lambda z^{-1}\right)^{-1}:|z|=1, z \neq 1\right\}$ are bounded and (ii) of Theorem 4.3 holds. We conclude that, for all $0<\lambda<1$, equation (4.15) has a solution in $\ell_{p}\left(\mathbb{N}_{0}\right)$. In [16, Section 7.1], an exhaustive analysis of the solutions of equation (4.15) when $f=0$ has been done which agrees with the condition on the parameter $\lambda$ provided by our abstract result.

In the next example, we provide an interesting criterion that ensures, under some assumptions on the operator $T$, the maximal $\ell_{p}$-regularity of equation (3.1).

Example 4.7. Let $X$ be a Hilbert space and $T \in \mathcal{B}(X)$ satisfying the following condition

$$
\| T||<w_{\alpha, \beta, \tau}:=\min _{|z|=1}\left|f_{\alpha, \beta, \tau}(z)\right|<1 \quad \text { where } \quad f_{\alpha, \beta, \tau}(z):=z^{1-\alpha}(z-1)^{\alpha}-\beta z^{-\tau} .
$$

By condition (C) and an application of the minimum principle, we obtain that $f_{\alpha, \beta, \tau}(z) \in \rho(T)$ and

$$
\left(f_{\alpha, \beta, \tau}(z)-T\right)^{-1}=\sum_{n=0}^{\infty} \frac{T^{n}}{\left(f_{\alpha, \beta, \tau}(z)\right)^{n+1}},
$$


whenever $|z| \leq 1$. Hence there exists a circle $\Gamma$ centered at the origin of radius $R<1$ such that $\sigma(T) \subset$ $\operatorname{Int}(\Gamma)$ and therefore

$$
S_{\alpha}(n):= \begin{cases}\frac{1}{2 \pi i} \int_{\Gamma} z^{n}\left(z^{1-\alpha}(z-1)^{\alpha}-\beta z^{-\tau}-T\right)^{-1} d z & n \in \mathbb{N}, \\ 0 & n=-\tau, \ldots, 0,\end{cases}
$$

defines an $\alpha^{\tau}$-resolvent family. Observe that by condition $(C)$ we have

$$
\left\|\left(f_{\alpha, \beta, \tau}(z)-T\right)^{-1}\right\| \leq \frac{1}{\left|f_{\alpha, \beta, \tau}(z)\right|-\|T\|}<\frac{1}{w_{\alpha, \beta, \tau}-\|T\|} .
$$

As a consequence, for all $n \in \mathbb{N}$, we have

$$
\left\|S_{\alpha}(n)\right\|<\frac{R^{n+1}}{w_{\alpha, \beta, \tau}-\|T\|}
$$

and then $\sup _{n \in \mathbb{N}}\left\|S_{\alpha}(n)\right\|<\infty$. Moreover,

$$
\sup _{|z|=1, z \neq 1}\left\|z^{1-\alpha}(z-1)^{\alpha}\left(f_{\alpha, \beta, \tau}(z)-T\right)^{-1}\right\|<\infty \quad \text { and } \sup _{|z|=1, z \neq 1}\left\|z^{-\tau}\left(f_{\alpha, \beta, \tau}(z)-T\right)^{-1}\right\|<\infty .
$$

By Theorem 4.3, we deduce that under condition (C) the following equation

$$
\Delta^{\alpha} u(n)=T u(n)+\beta u(n-\tau)+f(n),
$$

with homogeneus initial conditions $u(i)=0$, for all $i=-\tau, \ldots, 0$ has maximal $\ell_{p}$-regularity. Observe that for some values of $\alpha, \beta, \tau$ the only operator $T$ that satisfies condition (C) is $T=0$. See Figure 1 obtained with MATLAB.

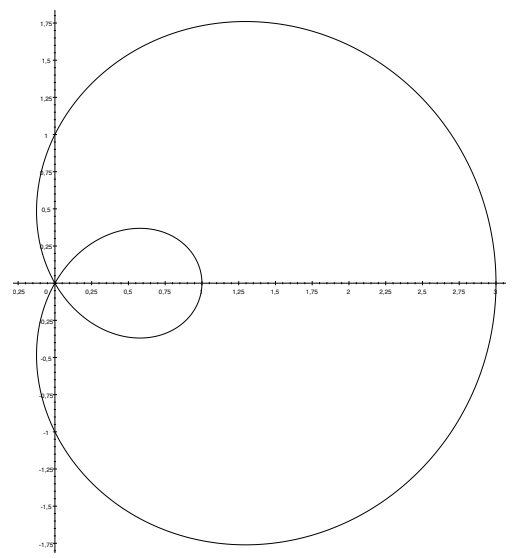

Figure 1: $f_{1,-1,2}(z), \quad|z|=1$

As an application of the criterion shown, we consider the equation

$$
\Delta u(n)=T u(n)-\lambda u(n-1)+f(n), \quad \lambda>0,
$$

where $\alpha=1, \beta=-\lambda$ and $\tau=1$. Then $f_{\alpha, \beta, \tau}(z)=f_{1,-\lambda, 1}(z)=z-1+\lambda z^{-1}$ and it can be easily computed that $\min _{|z|=1}\left|f_{1,-\lambda, 1}\right|=\lambda$. See the figure below. As a result, we get that for all $T \in \mathcal{B}(X)$ defined on a Hilbert space $X$ such that $\|T\|<\lambda<1$ equation (4.18) has maximal $\ell_{p}$-regularity. 


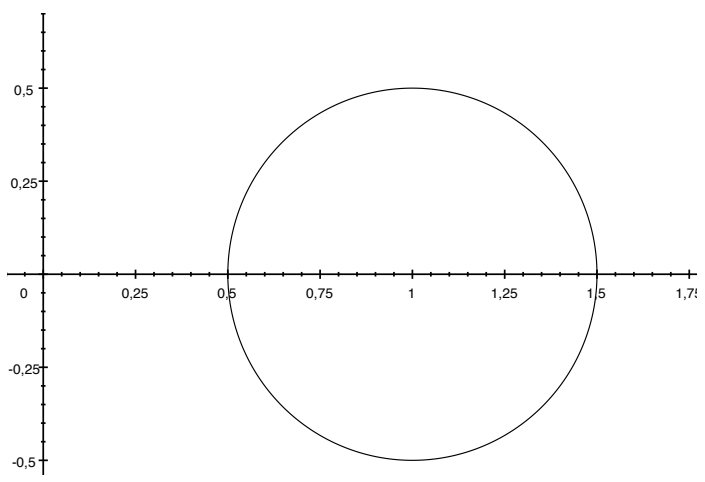

Figure 2: $f_{1,-0.5,1}(z), \quad|z|=1$

\section{References}

[1] R. P. Agarwal, C. Cuevas and C. Lizama. Regularity of Difference Equations on Banach Spaces, Springer, Cham, 2014.

[2] G. Akrivis, B. Li and C. Lubich. Combining maximal regularity and energy estimates for the discretizations of quasilinear parabolic equations. Math. of Comp. http://dx.doi.org/10.1090/mcom/3228.

[3] H. Amann. Linear and Quasilinear Parabolic Problems. Monographs in Mathematics, 89, Birkhäuser, Basel, 1995.

[4] W. Arendt and S. Bu. The operator-valued Marcinkiewicz multiplier theorem and maximal regularity. Math. Z., 240 (2002), 311-343.

[5] F. M. Atici and P. W. Eloe. A transform method in discrete fractional calculus. Int. J. Difference Equ., 2 (2) (2007), 165-176.

[6] F. M. Atici and P. W. Eloe. Discrete fractional calculus with the nabla operator. Electron. J. Qual. Theory Differ. Equ., 3 (2009), 1-12.

[7] H. Bateman. Some simple differential difference equations and the related functions. Bull. Amer. Math. Soc. 49, (1943), 494-512.

[8] A. Bátkai and S. Piazzera. Semigroups for delay equations. Research Notes in Mathematics, 10, Wellesley, MA, 2005.

[9] S. Blunck. Maximal regularity of discrete and continuous time evolution equations. Studia Math., 146 (2) (2001), 157-176.

[10] S. Bu. Mild well-posedness of equations with fractional derivative. Math. Nachr., 285 (2-3) (2012), 202-209.

[11] S. Bu. Well-posedness of fractional differential equations on vector-valued function spaces. Integral Equations Operator Theory, 71 (2) (2011), 259-274.

[12] S. Bu. Well-posedness of second order degenerate differential equations in vector-valued function spaces. Studia Math. 214 (1) (2013), 1-16.

[13] J.K. Hale and W. Huang. Global geometry of the stable regions for two delay differential equations. J. Math. Anal. Appl. 178 (1993), 344-362.

[14] R. Denk, M. Hieber and J. Prüss. R-boundedness, Fourier multipliers and problems of elliptic and parabolic type. Mem. Amer. Math. Soc., 166, 2003. 
[15] O. Diekmann, S. van Giles, S. Verduyn Lunel and H. Walter. Delay Equation. Springer, New York, 1995.

[16] S. Elaydi. An Introduction to Difference Equations. Springer, New-York, 2005.

[17] X. Fu, M. Li. Maximal regularity of second-order evolution equations with infinite delay in Banach spaces. Studia Math. 224 (3) (2014), 199-219.

[18] C. Hu and B. Li. Spatial dynamics for lattice difference equations with a shifting habitat. J. Differential Equations 259 (2015), 1957-1989.

[19] B. Kovács, B. Li and C. Lubich. A-stable time discretizations preserve maximal parabolic regularity. arXiv:1511.07823v1 [math.NA].

[20] B. Li. Maximum-norm stability and maximal $L^{p}$ regularity of FEMs for parabolic equations with Lipschitz continuous coefficients. Numer. Math. 131 (2015), 489-516.

[21] B. Li and W. Sun. Regularity of the diffusion-dispersion tensor and error analysis of Galerkin FEMs for a porous media flow. SIAM J. Numer. Anal., 53 (3) (2015), 1418-1437.

[22] C. Lizama. The Poisson distribution, abstract fractional difference equations, and stability. Proc. Amer. Math. Soc., DOI: https://doi.org/10.1090/proc/12895. To appear.

[23] C. Lizama. $\ell_{p}$-maximal regularity for fractional difference equations on UMD spaces. Math. Nach., 288 (17-18) (2015), 2079-2092.

[24] C. Lizama and M. Murillo-Arcila. $\ell_{p}$-maximal regularity for a class of fractional difference equations on UMD spaces: The case $1<\alpha \leq 2$. Banach J. Math. Anal., 11 (1) (2017), 188-206.

[25] C. Lizama and M. Murillo-Arcila. Well-Posedness for discrete time fractional models. Submitted.

[26] C. Lizama and M. P. Velasco. Weighted bounded solutions for a class of nonlinear fractional equations. Fract. Calc. Appl. Anal., 19 (4) (2016), 1010-1030.

[27] J. Mallet-Paret. The global structure of traveling waves in spatially discrete dynamical systems. J. Dyn. Diff. Eq. 11 (1) (1999), 49-126.

[28] K. S. Miller and B. Ross. Fractional difference calculus. In: Univalent functions, fractional calculus, and their applications, 1989, pp. 139-152.

[29] V. Poblete. Maximal regularity of second-order equations with delay. J. Differential Equations 246 (1) (2009), 261-276.

[30] R. Ponce. Hölder continuous solutions for fractional differential equations and maximal regularity. J. Differential Equations 255 (10) (2013), 3284-3304.

[31] P. Portal. Maximal regularity of evolution equations on discrete time scales. J. Math. Anal. Appl. 304 (1) (2005), 1-12.

[32] Y. N. Raffoul. Stability and periodicity in discrete delay equations. J. Math. Anal. Appl. 324 (2) (2006), 1356-1362.

[33] V. E. Tarasov. Fractional-order difference equations for physical lattices and some applications. J. Math. Phys. 56 (10) (2015), 1-19.

[34] V. E. Tarasov. Fractional Liouville equation on lattice phase-space. Phys. A 421 (2015), 330-342.

[35] L. Weis. Operator-valued Fourier multiplier theorems and maximal $L_{p}$-regularity. Math. Ann., 319 (2001) 735-758.

[36] G.C. Wu, D. Baleanu, Z.G. Deng and S.D. Zeng. Lattice fractional diffusion equation in terms of a Riesz-Caputo difference. Phys. A 438 (2015), 335-339. 
[37] G. C. Wu and D. Baleanu. Discrete chaos in fractional delayed logistic maps. Nonlinear Dynamics 80(4) (2016), 1697-1703.

[38] G. C. Wu, D. Baleanu and H-P. Xie. Riesz Riemann-Liouville difference on discrete domains. Chaos 26 (8) (2016), 084308, 5 pp.

[39] L. Xiao-Yan and J. Wei. Solving fractional difference equations using the Laplace transform method. Abstr. Appl. Anal., 2014 (2014), 1-6.

[40] Z. X. Yu. Uniqueness of critical traveling waves for nonlocal lattice equations with delays. Proc. Amer. Math. Soc. 140 (11) (2012), 3853-3859. 